\title{
Paediatric home care in Tower Hamlets: a working partnership with parents
}

\author{
Maybelle A Tatman, Caroline Woodroffe, Paula J Kelly, Roger J Harris
}

Wolfson Child Health Monitoring Unit, Division of Public Health: Epidemiology and Biostatistics Institute of Child Health, London WC1N 1EH

Maybelle A Tatman, research paediatrician Caroline Woodroffe, director

Tower Hamlets Paediatric Home Care Team, Steel's Lane Health Centre,

London E1 OLR

Paula J Kelly, team leader

Roger J Harris, consultant paediatrician

Correspondence and requests for reprints to: Dr Tatman

Accepted for publication 24 April 1992

\begin{abstract}
Objectives - To describe the first two years of a paediatric home care service.

Design - Observational cross sectional study, 1989-91.

Setting - One inner London health district.

Patients - 611 children referred to the service; 50 children selected from those referred during the first year, whose parents were interviewed and whose general practitioners were invited to complete a questionnaire.
\end{abstract}

Main measures - Description and costs of service; views of parents and general practitioners of selected sample of children.

Results - In its second year the team received 303 referrals and made 4004 visits at a salary cost of $£ 98000$, an average of $£ 323 /$ referral and $£ 24 /$ visit. This represented a referral rate of $3.2 \%$ (258/7939) of inpatient episodes from the main referring hospital between 1 December 1989 and 30 November 1990. Of all referrals to the service, $343(56 \%)$ came from hospital inpatient wards. The service was used by disadvantaged and ethnic minority families. The children's parents (in $28(61 \%$ ) families) and the home care team did a wide range of nursing tasks in the home. Parents of 47(94\%) children sampled agreed to be interviewed, and those of $43(91 \%)$ found the service useful; guidance and support were most commonly appreciated (33, $70 \%$ ). Parents of $25(53 \%)$ children said that hospital stay or attendance had been reduced or avoided. Parents and general practitioners disagreed on clinical responsibility in 10 children, and communication was a problem for some general practitioners.

Conclusions - The service enabled children to receive advanced nursing care at home. Clinical responsibility should be agreed between parents and professionals at referral.

(Quality in Health Care 1992;1:98-103)

\section{Introduction}

The Department of Health recommends that children should stay in hospital only if the care they need cannot be provided at home, in the outpatient department, or on a day basis in hospital. ${ }^{1}$ Paediatric home care offers an alternative to hospital, but it must provide an appropriate and coordinated service for children and adequately support their families. $^{2}$

We describe how far this was achieved in the first two years of a paediatric home care service in Tower Hamlets.

\section{BACKGROUND}

Tower Hamlets is in east London and has a Jarman underprivileged area score of $54.5,{ }^{3}$ the overall score for England and Wales being zero. The child population aged $0-14$ is $34582^{4} ; 40 \%$ of school children are from Bangladeshi families. ${ }^{3}$

Since April 1991 acute and community child health services have been provided by the Royal London Trust. The Queen Elizabeth Hospital for Children (Hospitals for Sick Children Special Health Authority), on the district boundary, provides further medical and surgical facilities and a casualty service. A few children attend hospitals outside the district.

In 1989 high rates of hospital admission among infants ${ }^{5}$ and the perceived difficulties of disadvantaged families in ethnic minorities in coping with the management of children in hospital led to the establishment of the paediatric home care team with funding for three years from the Tower Hamlets Inner Area Programme. The scheme was approved by the local community health council, family practitioner committee, and medical committee and by the local authority.

The first nurse appointed to the team (PJK) started in April 1989 and spent three months developing service objectives, recruiting other team members, and discussing the service with staff in hospital and in primary care.

AIM AND OBJECTIVES OF HOME CARE SERVICE The service was set up for sick children in Tower Hamlets whose nursing needs would normally be met in hospital. Its aim was to improve the quality of their care by extending the role of the sick children's nurse into the community. The main objectives were as follows:

- To be an alternative to hospital admission for sick children being referred by general practitioners and casualty and outpatient departments

- To shorten stay in hospital for admitted children

- To support the families of children admitted to hospital and increase their independence by enabling them to provide nursing care at home

- To provide an equitable service, accessible 
to disadvantaged families and giving families speaking Bengali informed choice in their child's management.

STRUCTURE OF HOME CARE TEAM

The team members were four full time registered sick children's nurses, a full time Bengali interpreter, and a part time secretary, based and managed in a health centre. The interpreter received training in counselling families with children with disabilities.

Referral criteria were not specific to particular diagnoses, except that children with diabetes continued to be managed by a specialist diabetes nurse who was not part of the home care team. Children who lived in Tower Hamlets and required practical nursing care at home or in school could be referred by any health professional. Each referral had to be agreed between the referrer and the parents, who were given the option of continuing with standard care. Hospital facilities remained available to children.

As this was a new service the home care team at first initiated referrals by discussion with hospital staff. As the service became more familiar, referrals were initiated by hospital and primary care staff and, to a small extent, by parents.

For each child referred visiting requirements were negotiated with parents, who were also able to request visits by telephoning the health centre. Members of the team visited during the day, seven days a week, and night time visiting was restricted to terminally ill children and to one child receiving overnight ventilation. The interpreter usually accompanied a nurse but on occasion went with families to hospital outpatient appointments. The team used the primary nursing model so that one nurse would be responsible for planning a child's care with the parents, enabling continuity to be maintained. Children were discharged when they no longer required nursing. Families could continue to be visited for counselling after their child had died.

Records of children referred and visits carried out were held by the team but clinical details were kept in records held by the parents.

While planning the service the consultant paediatrician $(\mathrm{RJH})$ invited comments from all general practitioners in Tower Hamlets, as a result of which it was agreed that they would be clinically responsible for the children at home.

The team maintained contact with staff at the Royal London Trust and the Queen Elizabeth Hospital. It was emphasised that in addition to the written notification of referral sent by the home care team to each child's general practitioner, hospital staff had a responsibility of discussing the children referred to home care with the children's general practitioners and health visitors before discharge.

AIM OF STUDY

The aim of the study was to examine how far

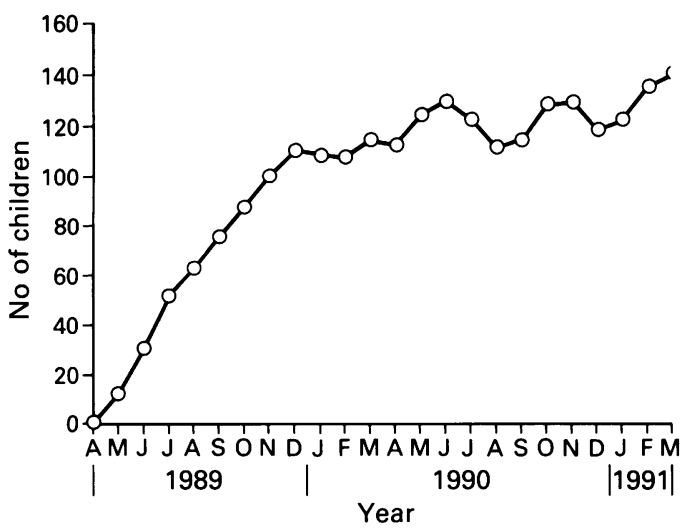

Caseload in first two years (active caseload at end of each month)

the objectives of the service were met, by describing the use of the service in its first two years and a survey of parents and general practitioners of a sample of children referred in the first year.

\section{Methods}

As part of the routine management of the service we recorded details of the referred children. They included age, diagnosis, language spoken by the family, date and source of referral, number of visits made by the nurses, and date of discharge from home care. We also monitored the caseload, comprising children being regularly visited by the team and children known to the team whose parents could request help.

To illustrate the scale of the service the total number of inpatient episodes in the Royal London Trust (then The London Hospital) for children in Tower Hamlets was compared with the number of children referred to the service from this hospital over one year. The starting date was 1 December 1989, when the caseload approached a stable level (figure).

The costs of the service were estimated from records kept by the team and excluded costs to families, other health services, or the local authority.

The work of the team was examined in detail by an observational cross sectional survey of a stratified sample of 50 children. The sample was drawn in May 1990 and weighted in proportion to the contribution of "low demand", "medium demand," and "high demand" children to the workload (table 1), as defined by the number of visits they had received at that time. "Low demand" patients were drawn from those referred after January 1990 to avoid problems with recall. At the time of the survey 20 of the children in the sample were still being seen by the home care team, 17 had been discharged within the previous two months, and 13 before then, including two children who had died.

The families of the children in the sample were interviewed at home by MAT with a list of open ended questions. Parents were informed that MAT was not a member of the home care team and that their comments would be anonymous. They were asked what they had to do to look after their child at home and how this compared with what they had to 
Table 1 Selection of study sample in May 1990 after first year of paediatric home care service

\begin{tabular}{lccccc}
\hline Patient group & $\begin{array}{c}\text { Visits to group by } \\
\text { May 1990 }\end{array}$ & $\begin{array}{c}\text { No of patients } \\
\text { in group }\end{array}$ & No excluded & Sampling fraction & No in study sample \\
\hline Low demand ( $\$ 10$ visits) & 885 & 239 & $\begin{array}{c}168 \text { referred before } \\
1 \text { January 1990 }\end{array}$ & 1 in 4 & 18 \\
Medium demand (11-30 visits) & 909 & 48 & 0 & 1 in 3 & 16 \\
High demand ( $\geqslant 31$ visits) & 860 & 16 & 0 & 168 & 16 \\
\hline Total & 2654 & 303 & in 1 & 50 \\
\hline
\end{tabular}

do in hospital, what the home care team had done and whether this had been useful, who they would contact if the child got worse, who else lived in the household, whether they or their partners were employed, and whether anyone else helped with child care or housework, and they were asked about their housing tenure. Families speaking Bengali were interviewed with a research interpreter who was not the one attached to the home care team.

Each child's general practitioner was asked by postal questionnaire whether he or she had received information about the child's referral and subsequent care, how useful the team was in the child's care, and whether the practice had a nurse who could have visited the child instead. Like the parents, general practitioners were asked who the parent should contact if the child deteriorated at home.

\section{Results}

FIRST TWO YEARS OF HOME CARE SERVICE

The caseload built up gradually in the first eight months (figure). In the first year, which ended on 30 April 1990, there were 303 children referred, 197 children discharged, and 2654 visits, with 106 children on the caseload at the end of the year. In the second year there were 308 children referred, 265 children discharged, 4004 visits, and 155 children on the caseload at the end of the year.

Of the 611 children referred, $165(27 \%)$ were aged under one year, $293(48 \%) 1-4$ years, $104(17 \%) 5-9$ years, and $49(8 \%)$ over
10 years. Children from families speaking Bengali were $270(44 \%)$ of the total and received a similar proportion of visits as the children from other families.

The London Hospital was the source of $483(79 \%)$ children, 306 of these being referred from the wards, 156 from outpatient clinics, and 21 from the accident and emergency department. Other hospitals referred $37(6 \%)$ children. Seventy three $(12 \%)$ children were referred by their general practitioner and $18(3 \%)$ by health visitors or school nurses.

Of 7939 episodes at The London Hospital between 1 December 1989 and 30 November 1990 in children resident in Tower Hamlets, $258(3 \cdot 2 \%)$ were associated with a new referral to the home care team.

Table 2 summarises the case types, nursing needs, and visiting requirements of children referred to the team

Table 3 shows the costs of the scheme. In the second year the average salary costs were $£ 24$ per visit and $£ 323$ per referral.

SAMPLE SURVEY OF CHILDREN REFERRED IN FIRST YEAR

The parents of $47(94 \%)$ of the sample of 50 children referred in the first year agreed to be interviewed, and the general practitioners of $36(72 \%)$ children returned their questionnaires.

Home circumstances of families

The 47 children lived in 46 families (two were siblings), of which all but four contained both

Table 2 Case types of children referred, care given, total visits, and mean visits per child for first two years of home care service

\begin{tabular}{|c|c|c|c|c|}
\hline Case type & Type of care & $\begin{array}{l}\text { Children referred } \\
(\%(\mathrm{No}))\end{array}$ & $\begin{array}{l}\text { Visits } \\
(\%(N o))\end{array}$ & $\begin{array}{l}\text { Mean } \\
\text { visits/child }\end{array}$ \\
\hline Dressings & Care of surgical wounds, burns, and scalds & $27 \cdot 0(165)$ & $15 \cdot 6(1039)$ & $6 \cdot 3$ \\
\hline Skin care & $\begin{array}{l}\text { Bathing, occlusive bandaging, mainly for severe } \\
\text { eczema }\end{array}$ & $15 \cdot 4(94)$ & $11 \cdot 0(732)$ & $7 \cdot 8$ \\
\hline Asthma - nebuliser & $\begin{array}{l}\text { Education on use of nebuliser and asthma } \\
\text { management }\end{array}$ & $10 \cdot 5(64)$ & $6 \cdot 9(459)$ & $7 \cdot 2$ \\
\hline Asthma - no nebuliser & $\begin{array}{l}\text { Education on asthma and checking child's inhaler } \\
\text { technique }\end{array}$ & $10 \cdot 2(62)$ & $2 \cdot 1(140)$ & $2 \cdot 2$ \\
\hline Other respiratory problems & $\begin{array}{l}\text { Low flow oxygen, tracheostomy care, cystic } \\
\text { fibrosis care }\end{array}$ & $4 \cdot 9(30)$ & $16 \cdot 3(1086)$ & $36 \cdot 2$ \\
\hline Giving drugs & $\begin{array}{l}\text { Growth hormone injections, helping drug } \\
\text { compliance }\end{array}$ & $4 \cdot 9(30)$ & $5 \cdot 0(333)$ & $11 \cdot 1$ \\
\hline Renal & $\begin{array}{l}\text { Blood pressure, weighing, collecting urine } \\
\text { specimens }\end{array}$ & $4 \cdot 4(27)$ & $9 \cdot 3(619)$ & $22 \cdot 9$ \\
\hline Special needs & Tube feeds, airway suction, controlling fits & $3 \cdot 9(24)$ & $4 \cdot 2(279)$ & $11 \cdot 7$ \\
\hline Prematurity & Daily weighing and monitoring, tube feeds & $2 \cdot 9(18)$ & $2 \cdot 8(186)$ & $10 \cdot 4$ \\
\hline Oncology & $\begin{array}{l}\text { Hickman line care, blood counts, some } \\
\text { chemotherapy, palliative care }\end{array}$ & $2 \cdot 1(13)$ & $8 \cdot 2(546)$ & $42 \cdot 0$ \\
\hline Haemoglobinopathy & $\begin{array}{l}\text { Desferrioxamine, blood tests, care of mild sickle } \\
\text { crisis }\end{array}$ & $1 \cdot 9(12)$ & $3 \cdot 6(240)$ & $20 \cdot 0$ \\
\hline Apnoea monitoring & Teaching use of alarm and how to respond & $1 \cdot 3(8)$ & $0 \cdot 5(33)$ & $4 \cdot 2$ \\
\hline Heart defect & Tube feeds, drugs, weighing, care postoperatively & $1 \cdot 1(7)$ & $3 \cdot 9(260)$ & $37 \cdot 1$ \\
\hline Other case types & $\begin{array}{l}\text { Various: traction, care of acute infections, eye } \\
\text { care, etc }\end{array}$ & $9 \cdot 4(57)$ & $10 \cdot 6(706)$ & $12 \cdot 4$ \\
\hline Total & & $100(611)$ & $100(6658)$ & $10 \cdot 9$ \\
\hline
\end{tabular}


Table 3 Costs $(£)$ of paediatric home care service

\begin{tabular}{lccr} 
& 1 April 1989- & 1 April 1990- & Total \\
31 March 1990 & 31 March 1991 & \\
\hline Salaries (pay with London weighting allowances and & 23 employers' costs): & \\
1 Nurse grade H & 214 & 24075 & 47289 \\
3 Nurses grade G & 41920 & 52722 & 94642 \\
Interpreter & 7292 & 13037 & 20329 \\
Clerical & 4324 & 7130 & 11454 \\
Agency nurse & 0 & 873 & 873 \\
Research interpreter & 0 & 250 & 250 \\
\hline Year subtotals & 76750 & 98087 & 174837 \\
\hline Capital equipment (depreciated over five years): & 1069 & 1069 & 2138 \\
Car & 300 & 300 & 600 \\
Computer & 221 & 221 & 442 \\
Furniture & 880 & 880 & 1760 \\
Medical equipment & 2470 & 2470 & 4940 \\
\hline Year subtotals & & & \\
\hline Recurring costs: & 192 & 192 & 384 \\
Bleeps & 184 & 376 & 360 \\
Telephone & 266 & 103 & 3500 \\
Stationery & 1500 & 2000 & 929 \\
Mileage & 328 & 601 & 5742 \\
Medical supplies & 2470 & 3272 & 185519 \\
\hline Year subtotals & 81690 & 103829 & \\
\hline Year totals & & & \\
\hline
\end{tabular}

parents. Eleven children had no siblings, 10 had one sibling, and 26 had more than one sibling, up to a maximum of seven. Twenty seven $(64 \%)$ of the 42 fathers and five $(11 \%)$ of the 46 mothers were employed. One father was in prison. Four families were owner occupiers; one rented privately; and the $41(89 \%)$ others, of whom three were in temporary accommodation, lived in local authority housing. Twenty two $(48 \%)$ of the families were Bangladeshi.

In thirteen $(28 \%)$ families someone other than the child had a long term illness. Five of the mothers were pregnant during the time their child had home care. Eleven families were helped by grandparents and a further six by other relatives. One family had a childminder and one had a local authority family aide.

\section{Nursing care given by parents}

Children's nursing needs ranged from one or two relatively simple tasks a day, such as changing dressings, to complex regimens of continuous oxygen, oximetry, nebulisers, tube feeds, and physiotherapy. Because the home care team provided visits rather than continuous nursing, children requiring frequent care were dependent on their parents. Fathers often helped, but the mother invariably took the main responsibility for care.

Nursing tasks were done by $28(61 \%)$ of the families and included giving drugs by nebuliser or injection, changing dressings, giving skin treatments, passing nasogastric tubes and giving nasogastric feeds, obtaining urine or blood specimens, giving low flow oxygen, chest physiotherapy, enterostomy or tracheostomy care, and hip traction. One child was ventilated at night. Tasks were demonstrated by the home care team and were then supervised and monitored, with the result that parents were confident. Parents tended to regard even demanding care as an extension of their parenting role: "I enjoyed caring for him - he was my life," and to take pride in their ability: "I was amazed at myself and at how quickly she healed." Regimens were incorporated into the home routine: "You slot it in - it becomes a way of life."

\section{Role of home care team}

The home care team provided practical nursing care to $27(57 \%)$ of the children, including changing dressings, skin care, venepuncture, giving injected drugs, and monitoring progress with treatment. Sixteen families $(34 \%)$ had relied on the nurses for medical supplies.

Parents of $43(91 \%)$ children had found home care useful. In addition to practical care, parents of $33(70 \%)$ thought that the guidance and support they had received were important, and those of $25(53 \%)$ were reassured by the nurses monitoring the child's condition. The families of four $(9 \%)$ children found home care of little use, either because the problem was minor or because the visits had not reduced their workload.

All of the 22 families who spoke Bengali had been visited by the home care team with their interpreter. When surveyed only three spoke English well enough to be interviewed without help from the research interpreter. Parents expressed dissatisfaction with provision of interpreting facilities in hospital, relying on English speaking relatives or older children for help. Many volunteered that full explanation of the child's management did not occur until the home care team visited.

\section{Comparison of hospital and home care}

Parents of $15(32 \%)$ children felt that hospital admission had been avoided or reduced by home care, parents of $10(22 \%)$ that day attendance at hospital had been avoided, those of $11(24 \%)$ that there had been no change, and those of $11(24 \%)$ did not know or said that it was impossible to tell.

Thirty five children (74\%) had previously been admitted to hospital, 20 of whom had had their parents resident with them; the other parents visited, 14 having been unable to stay overnight because of their other children and one because she had anxiety attacks in hospital. All parents felt that their children were happier at home than in hospital, but for eight their child's distress on admission was a particular problem. Ten mothers had carried out nursing tasks while in hospital, such as giving nebulisers, helping to tape drips, giving tube feeds, and changing traction. Thirteen of the 35 families were satisfied with hospital care, commenting that it offered security and helpful staff, but seven complained of the lack of facilities for parents to sleep beside their child's bed and five of a lack of parental control over the child's clinical management or daily routine. Seven parents had felt alienated or frightened by the hospital environment and three complained of the lack of privacy, one commenting: "When you are in hospital you are trying to live somewhere that is someone else's work space."

Considering these factors, 23 families felt that their child being in hospital was more 
Table 4 Opinions on clinical responsibility for child at home

General practitioners' opinion

General practitioner Hospital or home care team

Parents' opinion $\left\{\begin{array}{l}\text { General practitioner } \\ \text { Hospital or home can tcam }\end{array}\right.$ 13

stressful than their child being at home; 10 found both situations similar. Two families felt more anxious about home care, but one opted to continue because their child was distressed in hospital and the other because staying in hospital with their child meant difficulty in arranging child care for siblings.

\section{Clinical responsibility}

When parents and general practitioners were asked where parents should get help if their child's condition deteriorated at home, answers were obtained from both sources for $33(70 \%$ ) children (table 4$)$. For simplicity, the answers were divided into those mentioning the general practitioner as responsible, either alone or alongside home care or hospital, and those not mentioning the general practitioner.

There was agreement between the responses of families and general practitioners for 23 of the children, 13 of whom were felt to be the general practitioner's responsibility and 10 the responsibility of the hospital or home care team. Both general practitioners and parents felt that the children whose condition was less stable were the responsibility of the paediatricians. There was disagreement in 10 cases. General practitioners felt responsible for nine children, but the parents said that they had been told to go straight to hospital if the child deteriorated, that they preferred to contact the home care team, that it was more convenient to attend hospital, or that their relationship with their general practitioner had broken down. Although the remaining child was considered by the parents to be the general practitioner's responsibility, the general practitioner wrote: "The home care team largely communicated fairly well, but as I had little information from the hospital as to the overall plan I found dealing with arising problems difficult. It was never clear to me where the responsibilities lay. The parents required a lot of counselling, again a difficult task without adequate hospital letters."

\section{Views of general practitioners}

General practitioners saw communication as a problem. Adequate information had been received by the general practitioner both at referral and in subsequent feedback for $10(28 \%)$ of the 36 children whose general practitioners replied to the questionnaire. When asked to rate the role of the home care team in the children's care, general practitioners rated it "useful" or "very useful" for $11(31 \%)$ children and as "essential" for eight $(22 \%)$, but the general practitioners of $17(47 \%)$ children replied "not known."

In $18(50 \%)$ cases the general practitioner worked with a practice nurse and in three he or she felt that the practice nurse could have been involved. Other general practitioners commented that their nurses had no paediatric experience or were busy with health promotion or care of the elderly.

\section{Discussion}

A high response rate $(94 \%)$ was obtained from parents by interviewing them in their own homes, using an interpreter where appropriate. Families were positive about the service and confident about the care they were giving, regarding it as a natural part of their daily routine rather than as a burden.

The home care team thought that in addition to enabling children to be nursed at home the service gave parents more choice and therefore more control over the child's nursing care. This, together with the primary nursing model, allowed greater continuity of care than that provided in hospital. The interpreter found that she helped Bangladeshi parents to deal with both hospital and home care staff on a more equal footing.

WAS HOME CARE SERVICE AN EFFECIIVI ALTERNATIVE TO HOSPITAL CARE?

As in other areas ${ }^{6-9}$ the service clearly provided an alternative to hospital care by enabling tasks to be carried out which would usually require supervision in hospital. Half of the parents surveyed were certain that their children had been able to spend more time at home as a result.

Some referrals from casualty, outpatient, and primary care may have represented avoided admissions to hospital, but the numbers were small compared with the total number of children admitted. Because most referrals came from hospital wards any effect of home care on hospital use would probably be in reducing the length of stay rather than avoiding initial admission. The home care team were referred only a small proportion $(3 \%)$ of the children admitted to hospital, but this may have saved a larger proportion of hospital inpatient days by maintaining children with long term problems at home. Strict referral criteria and quantitative objectives were not laid down at the outset of the service because of the desire to respond flexibly to the needs of families. This made it difficult to measure the effect of the service on hospital admission.

\section{WAS SUPPORT ADEQUATE ANI) HQUITABIY} PROVIDED?

For most families the home care team had a clear role in supporting, teaching, and reassuring them. This enabled mothers to be the main provider of nursing care for their child. Despite the demands made on them mothers felt capable of coping with their child's needs and chose to remain at home because the alternative was the stress and inconvenience of hospital admission.

The home care service was accessible to disadvantaged families: the social circumstances of the families in the sample reflected the deprivation of this inner city area. Often support from the extended family was lacking. The physical, psychological, and 
financial burdens carried by these families require further study, and improvements in other methods of support including self help groups, social security attendance allowances, and the provision of home helps and home respite care must be explored. Other work showed that families do need quality respite care and demand a high standard of nursing if their child requires further hospital admission. ${ }^{10}$

The Bangladeshis of east London are a deprived group who may find it hard to make the best use of health services. ${ }^{5}$ Language difficulties must be overcome and cultural needs be accommodated for the provision of health care to be equitable with other groups. The families in this survey had a clear need for help with interpreting, which was not adequately met in hospital. The interpreter was therefore crucial for the home care of Bangladeshi children, enabling parents to understand and participate in their child's management.

\section{WAS THE SERVICE APPROPRIATE?}

The home care service was found to be appropriate and useful by most of the parents interviewed. Children referred to home care were likely to have been selected as having appropriate needs and having parents able to take on their clinical care. Parental satisfaction therefore reflects the appropriateness of referrals, and it does not follow that the service would be accepted by the parents of other children.

WAS THE SERVICE WELL COORDINATED?

Good use of the resources of primary care depends on coordination with the home care team and with hospital care, demanding effective liaison. Despite the initial policy the children's general practitioners were not always given clinical responsibility for children receiving home care, and in some cases parents and general practitioners differed in their opinions of who was responsible.

FEEDBACK OF RESULTS TO STAFF

The results of the survey were discussed at a meeting with the consultant paediatrician and home care team to which all general practitioners in Tower Hamlets were invited. It was felt that the condition of some children receiving home care was sufficiently unstable to warrant open access to hospital and that the issue of clinical responsibility was determined by the needs of the child. Negotiation of this with parents at the outset of home care depended on good communication between paediatricians, primary health care teams, and home care nurses. In particular, paediatricians were responsible for handing over clinical responsibility to the general practitioner. Telephone calls were seen as the best but also as the most time consuming way of achieving this. Greater use of records held by the parents was also agreed.

In our experience paediatric home care improves the quality of some aspects of care, extends the choices available to families, and enables partnership with parents to meet the needs of children. Further work is necessary to improve liaison with the primary health care team. Just as the families slotted their child's health care into the rest of their lives, we need to think beyond our traditional models of provision to address more fully these families' needs.

We thank Saleha Uddin for interpreting, the Medical Research Council for funding the health services research training fellowship held by MAT, the Tower Hamlets Inner Area Programme for funding the home care service, the Tower Hamlets District Information Unit for providing the information on hospital episodes, the Wolfson Foundations for funding the Wolfson Child Health Monitoring Unit, and the parents and general practitioners who participated in the survey.

1 Department of Health. Welfare of children and young people in hospital. HMSO: London, 1991

2 Lessing D, Tatman MA. Paediatric home care in the 1990s. Arch Dis Child 1991;66:994-6.

3 Department of Community Medicine and Public Health, Tower Hamlets Health Authority. Tower Hamlets People. London: Tower Hamlets Health Authority, 1989. (Health report No 2.)

4 Central Statistical Office. Regional trends 26. London: HMSO, 1991.

5 Watson E. Health of infants and use of health services by mothers of different ethnic groups in East London. Community Med 1984;6:127-35.

6 Atwell JD, Gow MA. Paediatric trained district nurse in the community: expensive luxury or economic necessity? BMF 1985;291:227-9.

7 Glucksman E, Tachakra SS, Pigott S, Lea H. Home care team in accident and emergency. Arch Dis Child 1986;61:294-6.

8 Couriel JM, Davies P. Costs and benefits of a community special care baby service. BMF 1988;296:1043-6.

9 Whiting $M$. Community paediatric nursing in England in 1988 [MSc Thesis]. London: University of London, 1988: $145 \mathrm{pp}$.

10 Thornes R. Care of dying children and their families. Birmingham: National Association of Health Authorities, 1988 . 